


\title{
Implementación de una plataforma de computación en la Nube bajo el modelo de la infraestructura como servicio para la Universidad Industrial de Santander.
}

\section{Implementation of an iCloud Platform Based on Infrastructure- as- Service- Model for the Universidad Industrial de Santander.}

\author{
Emmanuell Díaz Carreño ${ }^{2}$, Manuel Flórez Becerra ${ }^{3}$ \\ ${ }^{1}$ Universidad Industrial de Santander, Colombia.
}

Artículo recibido en Julio de 2013; artículo aceptado en Octubre de 2013.

Citación del artículo: Díaz, E. \& Flórez, M. (2013). Implementación de una plataforma de computación en la Nube bajo el modelo de la infraestructura como servicio para la Universidad Industrial de Santander. I+D Revista de Investigaciones, 2(2), 49-60.

\section{Resumen}

La infraestructura de cómputo presente en entornos académicos y las limitaciones en la cantidad de los recursos con los que dichos entornos cuentan, hacen que sea necesario buscar formas de aprovechar la capacidad de hardware no utilizada; ante esta situación, la implementación de un modelo de servicios basado en el paradigma de la computación en la nube, representa una solución que no debe ser desestimada. Este trabajo de investigación presenta el proceso de implementación de un modelo de prestación de servicios, para apoyar los proyectos desarrollados por los miembros de la Escuela de Ingeniería de Sistemas e Informática de la Universidad Industrial de Santander (EISI), utilizando el modelo de computación en la nube conocido como IaaS o Infrastructure as a Service.

\footnotetext{
${ }^{1}$ Artículo de investigación tecnológica, de enfoque cuantitativo, resultado parcial de un proyecto de investigación en desarrollo denominado: Objeto Virtual de Aprendizaje para inglés de primer grado de educación básica primaria, desarrollado en el grupo de investigación en nuevas tecnologías aplicadas a la educación GIDSAW de la Corporación Universitaria de Investigación y Desarrollo (UDI) de la ciudad de Bucaramanga (Colombia). Calle 9 No.23-55, PBX:6352525. La investigación fue financiada por la misma institución universitaria.

${ }^{2}$ Ingeniero de Sistemas, Universidad Industrial de Santander. Docente-investigador del grupo de investigación y desarrollo en la Nube, seguridad, software y servicios. Universidad Industrial de Santander de la ciudad de Bucaramanga (Colombia).Correo electrónico:emmanuell.diaz@correo.uis.edu.co

${ }^{3}$ Ingeniero de Sistemas, Universidad Industrial de Santander. Magíster en sistemas, Universidad Industrial de Santander. Docente-investigador del grupo de investigación y desarrollo en la Nube, seguridad, software y servicios. Universidad Industrial de Santander de la ciudad de Bucaramanga(Colombia).Correo electrónico: mgflorez@uis.edu.co
} 
Palabras clave: Computación en la Nube, Infraestructura como Servicio, Software Libre.

\section{Abstract}

Computing infrastructure in academic environments and the resources limitations into them, make necessary looking for profit ways to take advantage of their useless hardware capacity; faced with it, the implementation of a service model based on the Cloud computing system is a solution to be taken into account. This research shows the application of a provision service model to support the projects developed by the members of the Systems Engineer and Information Technology School at the Universidad Industrial de Santander (EISI), using the Cloud computing model called IaaS or Infrastructure as a Service.

Keywords: Cloud computing, Infrastructure as service, free software.

\section{Introducción}

La computación en la nube se ha convertido en un concepto muy importante en los últimos años (Gartner, 2011); éste representa un cambio en la forma como se utilizan los centros de datos y un cambio de paradigma en desarrollo acerca de la computación y la manera como se usa y se accede a ésta (Maggiani, 2009).

Los modelos de prestación de servicios apoyados en la computación en la nube, han generado una industria que mueve miles de millones de dólares al año y a pesar de ser un paradigma con un par de años en el mercado, prácticamente considerado en su infancia, se ha posicionado con sus prestaciones, alta usabilidad y la posibilidad de usar servicios bajo demanda, como una de las tecnologías más prometedoras a corto plazo (Forrester Research, 2011).

El campo de la computación en la nube, ha sido investigado por la academia desde sus inicios, pero debido a la etapa en la cual se encuentra, en la literatura existente es fácil hallar información contradictoria acerca de los conceptos fundamentales que lo rodean (Geelan, 2011); a pesar de eso, hay cada vez un mayor consenso en dichos conceptos, gracias al gran potencial futuro de esta tecnología y a los esfuerzos realizados por desarrollar estándares por parte de múltiples entidades.

Las soluciones de código abierto presentan una oportunidad para que organizaciones interesadas en experimentar con la computación en la nube puedan hacerlo, sin necesidad de grandes inversiones iniciales de capital, ni infraestructura, ya que pueden hacer uso de sus equipos existentes y determinar cuáles son sus necesidades respecto a este nuevo paradigma.

Al inicio de esta investigación, la Escuela de Ingeniería de Sistemas e Informática de la Universidad Industrial de Santander (EISI), no contaba con un servicio de Computación en la Nube sobre la cual se pudiese realizar desarrollo de aplicaciones o la prestación de servicios. Además, su infraestructura poseía ineficiencias debido a la baja tasa de utilización de los recursos como son cómputo y almacenamiento y la dificultad de los miembros de la comunidad para hacer uso de dichos recursos. Debido a la 
adquisición de nuevo hardware por parte de la EISI, se hizo necesario el desarrollo de una plataforma sobre la cual se pudieran apoyar los diferentes grupos y miembros de la misma, para maximizar la utilización de dichos recursos y sobre la cual puedan desarrollar, implementar, probar y mejorar sus aplicaciones o proyectos, antes de ser presentados o publicados ante los interesados.

En este orden de ideas, surge la pregunta que orientó el presente trabajo de investigación tecnológica: ¿Qué dispositivo en la Nube y bajo qué modelo, puede permitir el desarrollo de aplicaciones y el suministro de servicios de cómputo y almacenamiento para la comunidad universitaria de la Universidad Industrial de Santander?

\section{Método}

\section{Tipo de estudio}

Se siguió un tipo de investigación tecnológica aplicativa, de nivel evaluativo, sobre el funcionamiento óptimo de una plataforma de computación en la Nube bajo el modelo de la infraestructura como servicio (IaaS).

\section{Participantes}

Docentes a cargo del proyecto y personal asistente de la Escuela de Ingeniería de la Universidad Industrial de Santander.

\section{Materiales e instrumentos}

El hardware sobre el cual se implementó la plataforma, consta de dos servidores Dell PowerEdge R720®, cada uno con un procesador Xeon E5640® de ocho núcleos, 24 GB de RAM, un puerto Gigabit de conexión entre los dos equipos y un enlace de $100 \mathrm{Mbs}$ a la red local de la Universidad.

\section{sistema operativo.}

Se usó el sistema operativo Debian ${ }^{\circledR}$, versión Squeeze ${ }^{\circledR}$. La selección de dicho sistema operativo se da principalmente por la estabilidad asociada a los sistemas Debian ${ }^{\circledR}$ (Barbosa \& Muñoz, 2006).

\section{hipervisor}

En el proceso inicial, fue necesario seleccionar un hipervisor que cumpliera con los requisitos; se seleccionaron para análisis los hipervisores: Xen ${ }^{\circledR}, K V M \AA$, Virtualbox $\AA$, Hyper-V ${ }^{\circledR}$ y VMware ${ }^{\circledR}$. Estos fueron seleccionados al ser los que poseen mayor penetración en el mercado (Cloud Computing State of the Union, 2010).

Se seleccionó Virtualbox ${ }^{\circledR}$, dando mayor prioridad a su facilidad de manejo y de generación de servicios virtualizados por parte de los usuarios objetivo de esta investigación; con esto se buscó que la curva de adopción inicial del servicio fuese menos acentuada, y permitiera a futuro proveer un uso mayor de la plataforma. Virtualbox ${ }^{\circledR}$ permite que un usuario sin conocimientos técnicos elevados transforme su máquina virtual en un servicio virtualizado; la simplicidad de dicha operación permite que los usuarios puedan realizar pruebas técnicas locales y luego de estar conformes con sus resultados, puedan alojar estas instancias en el servicio de computación en la nube, para continuar su trabajo desde ahí. Respecto a la licencia de uso de Virtualbox, siendo tipo GPLv2® en su versión $O S E \circledast$, para la presente investigación se usó la 
versión directa de los binarios, que posee licencia PUEL (C) (Virtualbox, 2011) la cual permite su uso en entornos académicos para múltiples usuarios y diversos usos sin ningún costo. Este hipervisor no está limitado de ninguna manera a la cantidad de memoria RAM, el número de CPUs o núcleos que tenga el sistema, y tampoco limita el número de instancias virtuales que pueden estar activas en el sistema, como sí ocurre en algunos casos con los hipervisores Xen ${ }^{\circledR}$ o KVM® (Díaz, 2012). La compatibilidad con Debian ${ }^{\circledR}$ es completa y permite también emular arquitecturas x 86 y x86-64 sin inconvenientes.

\section{administrador IaaS}

Para el proceso de selección de una herramienta de administración, se estableció un requisito extra debido a la selección del hipervisor: dicha herramienta debe ser compatible con Virtualbox ${ }^{\circledR}$. Una selección de herramientas candidatas, y su posterior análisis (Díaz, 2012) permitió, con base en las características principales de cada una, determinar aquellas que debían ser descartadas. Consiste en un juego que tiene una secuencia básica que sigue los procesos de la manera como se muestra en la figura 1. Se realizó el proceso de instalación de las herramientas aún no descartadas, se analizó la complejidad para la instalación de cada una, esto pensando en las labores administrativas a futuro, al realizar migraciones del servidor o actualizaciones de la plataforma; se determinó también el grado de dificultad para la administración de los recursos, o para la supervisión de éstos. Este tipo de análisis representa una verdadera dificultad para realizar la selección (Peter, 2010) ya que se pasa de una selección basada en características comparables o medibles, al plano de las prestaciones de la herramienta, tanto las que ya están desarrolladas, como las que aparecen en los planes de desarrollo; este proceso eleva la complejidad de la selección y hace que se determine una característica a la cual darle mayor prioridad; en este caso, dicha característica fue la posibilidad de migrar las instancias virtuales, buscando aprovechar de mejor manera los recursos con que se cuenta. Con esta característica prioritaria y debido a que se busca que la plataforma construida cuente con una alta capacidad de expansión a futuro, se seleccionó OpenNebula ${ }^{\circledR}$ (OpenNebula, 2011). Otra de las características que motivaron la selección de OpenNebula ${ }^{\circledR}$, fue la modularidad de sus componentes; esta funcionalidad permite que a futuro se puedan usar otros hipervisores sin inconvenientes, y facilita el desarrollo de scripts (OpenNebula, 2011) que permitan agregar nuevas funcionalidades a la herramienta, sin necesidad de alterar el código fuente y sin quedar limitados al desarrollo que realicen los autores de la herramienta, además de las prestaciones para la construcción de una nube privada (Shader, 2010) y su compatibilidad con Occi Service Configuration ${ }^{\circledR}(\mathrm{CCI}, 2011)$ y con Open Cloud Computing Interface ${ }^{\circledR}$ (OCI, 2011); ésta es un conjunto de especificaciones desarrolladas por comunidades abiertas y reguladas a través del Open Grid Forum ${ }^{\circledR}$, que define un estándar para que los proveedores de servicios puedan prestar sus ofertas de cómputo, datos y redes. Otra ventaja con la que cuenta OpenNebula ${ }^{\circledR}$ es la reciente inclusión de una interfaz gráfica de administración y de usuario final, características que se pueden considerar clave, ya que toda la administración del servidor debe realizarse de manera remota, y además permite que el proceso de formación a nuevos administradores requiera 
menos tiempo; la interfaz de usuario final permite que los mismos usuarios administren, instancien y liberen recursos de acuerdo con sus necesidades y acuerdos de servicio.

\section{Procedimiento}

Para la realización del estudio se llevaron a cabo los siguientes pasos:

1. Se realizó un análisis de las necesidades de apoyo de los proyectos de la Escuela de Ingeniería (Díaz, 2012); a partir de los resultados de dicho análisis, se inició la construcción de una plataforma de computación en la nube basada en un modelo de infraestructura como servicio.

2. Los servicios que se determinaron para la EISI bajo el modelo de IaaS están ligados a ciertos parámetros que corresponden a la organización y estado de la misma; entre éstos se estableció la necesidad de asegurar que las herramientas de desarrollo que fueran ofrecidas, tuvieran un alto grado de seguridad, con el fin de preservar la confidencialidad de los proyectos; otra limitación fue determinar de qué manera se permitiría un acceso responsable y mesurado a los recursos asignados a cada uno de los beneficiarios de los servicios y cómo permitir que estos pudiesen disponer a la medida de sus necesidades, de las facilidades de migración o aprovisionamiento de recursos, sin afectar las labores de otros equipos de trabajo. Estas limitaciones son aún más marcadas cuando se trata de trabajo de grupos de investigación, en los cuales pueden presentarse necesidades de servicios de mayor capacidad, basados en sus diferentes proyectos.

3. Se definieron los parámetros de seguridad: (a) aislar la red virtualizada de la red de la universidad, es decir, sólo se permite acceso a los servicios solicitados por cada una de las instancias y así mismo solo estos servicios tienen acceso a la red externa; (b) automatizar las labores de detección de intrusos y generar alertas administrativas en los casos en que sea necesario, (c) generar automáticamente resúmenes de registros del sistema y de eventos anómalos, (d) permitir la sincronización de la hora de todas las instancias virtuales por medio del frontend, para permitir la realización de tareas administrativas programadas, (e) facilitar la administración de redes virtuales con la implementación de un servicio en el frontend, (f) ejecutar tareas de copias de seguridad remotas de la configuración del sistema, las bases de datos y los scripts de mantenimiento.

4. Se estableció el acceso a los usuarios, determinado por el tipo de instancia utilizada, es decir, un usuario que necesite una instancia virtual de un servidor de base de datos, solo tendrá acceso a su instancia por medio de los puertos necesarios para uso y administración; mientras que un usuario que tenga una instancia virtual de un servidor web, necesitará un puerto para acceso no seguro, otro para acceso seguro, un puerto para administración y un puerto para alojar archivos; sin embargo, el hecho de que una instancia virtual tenga definidos los puertos que usará, no significa que si un usuario requiere por algún caso en particular acceso a otro puerto no pueda ser otorgado.

5. Se analizó la seguridad de servidores, la cual puede verse comprometida por muchas causas, pero se puede esperar que una de las mayores causas sea por la inexperiencia en administración de este tipo de equipos (aún los 
virtuales); es por esto que el grupo CONUSS puso a disposición de los usuarios de la plataforma sus conocimientos en estas labores, como parte fundamental del proceso de adopción de este paradigma de cómputo para la EISI.

6. Se construyó el prototipo. En el proceso de construcción, se realizó un análisis de las herramientas necesarias para implementar un servicio de computación en la nube que cumpliese con los parámetros determinados en la fase de análisis y que además de esto cumpliera con otros requisitos que se plantearon desde el inicio de la investigación: (a) todas las herramientas usadas deben ser gratuitas, (b) no debe existir incompatibilidad de licencias o limitaciones de uso de las herramientas, (c) deben funcionar sobre el sistema operativo Debian. La gratuidad de las herramientas se requiere en proyectos netamente académicos, en los cuales se busca que puedan ser replicados sin necesidad de incurrir en costos iniciales, principalmente por licencias de sistema operativo o de aplicaciones; así mismo las aplicaciones que hacen parte de la plataforma no deben estar amparadas en licencias que no permitan el uso por más de un usuario o que estén limitadas a condiciones de hardware o software en el equipo. Finalmente, esta investigación se desarrolló paralelamente a un proyecto de administración de servidores (Barbosa \& Muñoz, 2010) del grupo de investigación CONUSS, en el cual se definió el sistema operativo de los nodos; éste delimitó la compatibilidad de las herramientas seleccionadas y a su vez definió una base segura sobre la cual construir el prototipo.

El prototipo implementado se compone de tres partes esenciales: un servidor principal o frontend que permite centralizar los servicios prestados y que actúa como supervisor entre los servicios ofrecidos, una herramienta de administración de la plataforma que permite orquestar los servicios prestados y un hipervisor que permite administrar todo el ciclo de vida de las instancias virtuales.

Con estos requisitos presentes, se realizó un proceso de investigación y comparación de aplicaciones candidatas y posteriormente su selección.

\section{Resultados}

\section{Carga máxima soportada}

Como base para calcular el máximo de instancias concurrentes, se establecieron las diferentes configuraciones del hardware virtualizado con las cuales trabajaría la plataforma, esto con el fin de permitir la mayor cantidad de usuarios compartiendo el mismo hardware, sin comprometer la estabilidad del equipo; las diferentes plantillas creadas para este fin, se presentan en la tabla 1.

Tabla1. Tipos de plantillas utilizadas.

\begin{tabular}{cccc}
\hline $\begin{array}{c}\text { Nombre } \\
\text { Plantilla }\end{array}$ & $\begin{array}{c}\text { Tamaño } \\
\text { Memoria }\end{array}$ & $\begin{array}{c}\text { Capacidad } \\
\text { HDD }\end{array}$ & $\begin{array}{c}\text { Capacidad } \\
\text { CPU }\end{array}$ \\
\hline small & $256 \mathrm{MB}$ & $10 \mathrm{~GB}$ & 0,25 Núcleos \\
medium & $512 \mathrm{MB}$ & $20 \mathrm{~GB}$ & 0,33 Núcleos \\
large & $756 \mathrm{MB}$ & $20 \mathrm{~GB}$ & 0,50 Núcleos
\end{tabular}

Fuente: Autor. 


\section{Uso de CPU}

En la Figura 1, se observa una representación del uso de la CPU durante la prueba, y su comportamiento a medida que se instanciaron máquinas de tipo small; el procedimiento realizado fue la instanciación de 5 máquinas virtuales cada 5 minutos, hasta llegar a un punto superior al 75\% del uso máximo de la CPU, y posteriormente se instanció cada 3 minutos una máquina extra, hasta superar el $80 \%$. El eje vertical representa el porcentaje del uso de la CPU y el eje horizontal, un periodo de 10 horas, desde las 00:00 hasta las 10:00, las pruebas inician a partir de las 03:00, se muestra un periodo más largo a las 7 horas de duración de la prueba para contrastar con el uso del equipo en estado ocioso.

Figura1. Uso de la CPU durante la prueba.

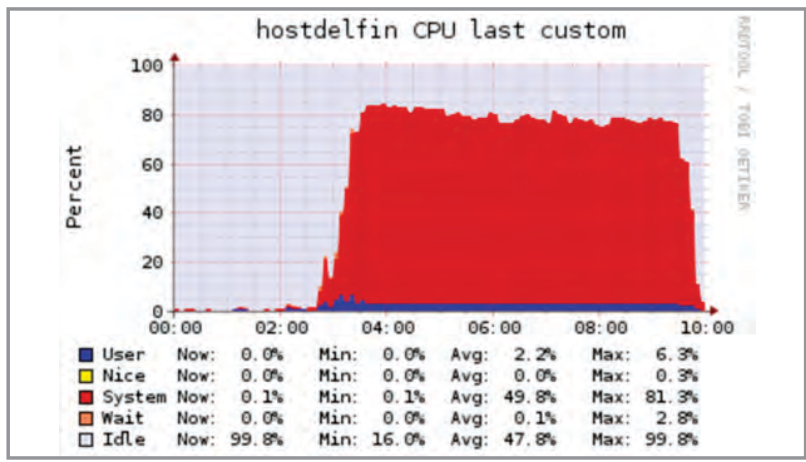

Fuente: Autor.

Al llegar a 34 máquinas virtuales como está representado por el eje vertical en la figura 2, se comprobó que se podía llegar hasta el límite teórico luego de este punto, y observar que la carga de CPU se mantuvo; se realizaron pruebas de conexión y respuesta del host (equipo en cual se crean y se ejecutan las máquinas), esto con el fin de comprobar que no se había perdido control sobre el equipo. Los resultados muestran que el host alcanzó un máximo de $81.3 \%$ de uso, y según los registros del sistema, un promedio de $64.9 \%$ durante las 7 horas de la prueba.

Fig. 2 Número de máquinas virtuales instanciadas durante la prueba

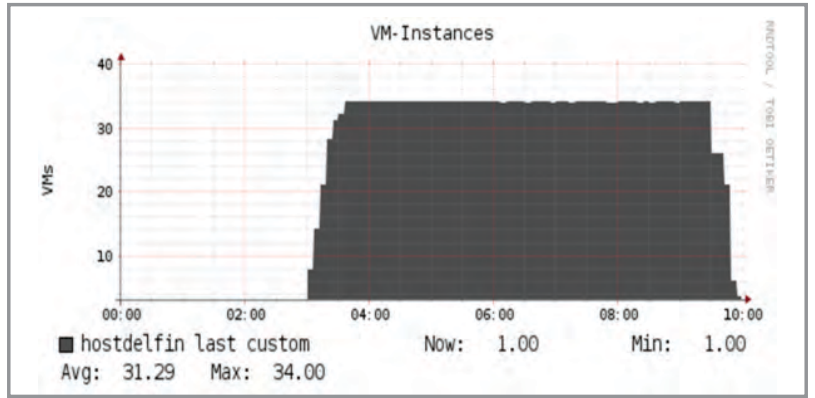

Fuente: Autor.

\section{Uso de memoria RAM}

El uso esperado de la memoria RAM durante la prueba realizada fue de $8.5 \mathrm{~GB}$, esto al tener 34 instancias en ejecución, es decir 34 VM x 0,25 $\mathrm{GB} / \mathrm{VM}=8.5 \mathrm{~GB}$. En laFig. 3, en el eje vertical se encuentra expresado en giga bytes el comportamiento del uso de la RAM durante las pruebas, el uso de la memoria aparece como máximo $8.8 \mathrm{~GB}$, lo cual concuerda con el valor calculado. (ver figura 3 ).

Figura 3. Uso de la memoria RAM durante la prueba.

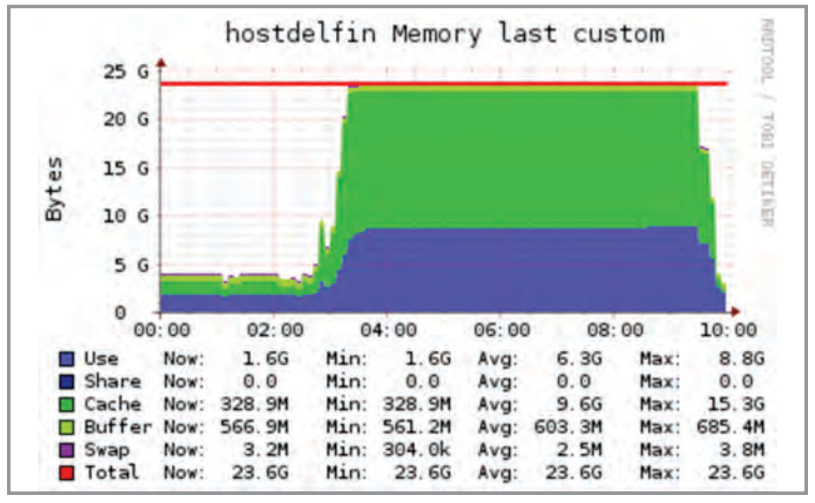

Fuente.Autor. 


\section{Consumo de energía}

Otro factor que se consideró importante analizar, fue la potencia total consumida por el sistema; se configuraron los servidores host para obtener este valor y así poder hacer estimaciones del grado de eficiencia del modelo; la figura 4 muestra el comportamiento del consumo de energía durante la prueba; en ésta, el eje vertical representa el consumo en vatios-hora del host.

Fig. 4 Potencia consumida por el sistema durante la prueba.

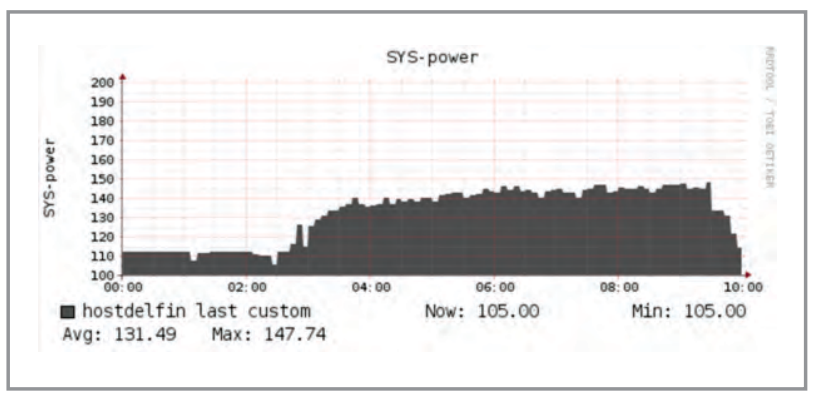

Fuente: Autor.

El host en estado de uso mínimo tiene un consumo eléctrico de $105 \mathrm{Wh}$; con las 34 máquinas instanciadas anteriormente, el consumo aumenta $147.74 \mathrm{Wh}$; esto quiere decir que el sistema está usando 42.74 Wh de más para ejecutar las 34 máquinas virtuales, lo cual equivale a $1.25 \mathrm{Wh}$ por cada máquina instanciada. El consumo generado por tener 34 equipos reales funcionando para prestar los servicios que se simularon, se puede aproximar de la siguiente manera: la plantilla utilizada corresponde a una instancia, usando 0,25 núcleos de la máquina real, esto es igual a un procesador de $667 \mathrm{MHz}$, un procesador similar en frecuencia de reloj (Ark ${ }^{\circledR}$, Intel ${ }^{\circledR}$, Pentium $\AA$, 2012) tiene un consumo de $17.5 \mathrm{Wh}$, esto equivale a un consumo de 595 Wh únicamente en procesador, es decir sin tener en cuenta los demás componentes de un equipo real; simulando el consumo de un equipo real con este tipo de procesador y usando una herramienta para el cálculo aproximado del consumo de energía (eXtreme Power Supply Calculator ${ }^{\circledR}, 2012$ ), se obtiene un valor aproximado de $86 \mathrm{Wh}$, es decir $2856 \mathrm{Wh}$ para 34 equipos equivalentes a los simulados, aproximadamente 19.36 veces más; ahora, si en lugar de ser máquinas con tan limitada capacidad, estas máquinas correspondieran a equipos con características similares al host, con un consumo de $105 \mathrm{Wh}$, el consumo se eleva a $3570 \mathrm{Wh}$ para tener a 34 equipos reales en funcionamiento, un consumo 24.20 veces mayor.

Trasladando estos resultados a costo monetario para un periodo de un mes y usando como precio de KWh el correspondiente al mes de febrero de 2012 (ESSAC, 2012) por parte del prestador del servicio, para el nivel de tensión que posee el edificio de tecnologías CENTIC (nivel II), el cual tuvo un valor de $\$ 341.32 / K W h$, y tomando un mes de 30 días, equivalente a 720 horas, se obtienen los siguientes valores:

\section{escenario 1.}

1 Servidor virtualizando 34 máquinas $1 * 0.148 \mathrm{~kW} / \mathrm{h} * 720=106.37 \mathrm{kWh} / \mathrm{mes}$ $106.37 \mathrm{kWh} / \mathrm{mes} * \$ 341.32 / \mathrm{kWh}=\$ 36.307 .45 /$ mes

escenario 2.

34 equipos de bajas especificaciones $34 * 0.086 \mathrm{kWh} * 720=2105.28 \mathrm{kWh} / \mathrm{mes}$ $2105.28 \mathrm{kWh} / \mathrm{mes} * \$ 341.32 / \mathrm{kWh}=$ $\$ 718.579 .85 / \mathrm{mes}$ 


\section{escenario 3.}

34 equipos de especificaciones similares al host $34 * 0.105 \mathrm{kWh} * 720=2570.40 \mathrm{kWh} / \mathrm{mes}$ $2570.40 \mathrm{kWh} / \mathrm{mes} * \$ 341.32 / \mathrm{kWh}=$ $\$ 877.335 .87 / \mathrm{mes}$

En la figura 5 se agruparon los costos calculados para comparar los valores obtenidos; cabe anotar que entre los costos energéticos también se deben incluir costos externos, como son en este caso los costos de refrigeración del lugar; por otra parte, el espacio ocupado por dichos equipos amplía el espacio a climatizar, a razón de cantidad de éstos; por consiguiente, el consumo de energía aumenta aún más en los escenarios en los cuales se contempla un mayor número de equipos reales.

Figura. 5. Costo energético aproximado.

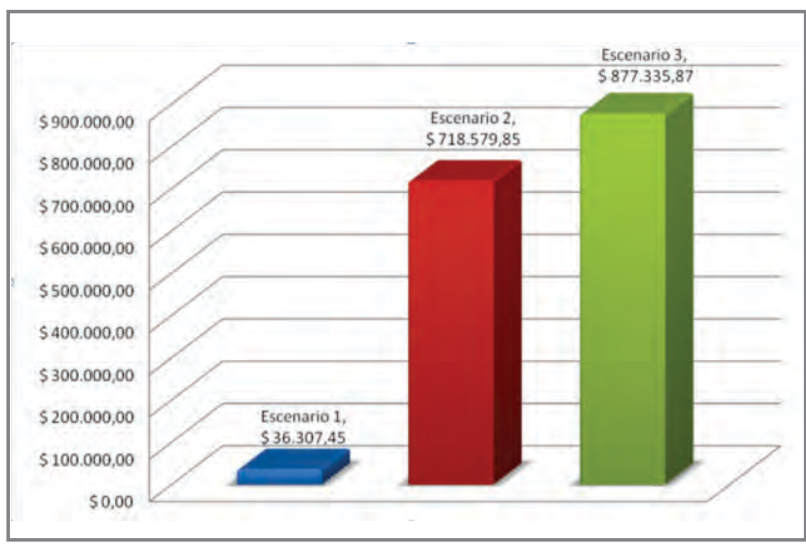

Fuente: Autor.

\section{Discusión}

El desarrollo de una plataforma de prestación de servicios de computación en la nube para comunidades académicas, usando soluciones de software libre o abierto, representa una gran oportunidad para el aprovechamiento eficiente de los recursos subutilizados con los que éstas cuentan, sin costos de inversión o de licenciamiento. A partir de las pruebas realizadas, se comprobó que la utilización eficiente de los recursos de cómputo con que cuenta la EISI, permite además de conseguir prestar servicios a un mayor número de miembros de la comunidad, la posibilidad de hacerlo con un menor costo energético, causando así una menor huella de carbono en el ambiente.

El cálculo de la carga máxima soportada por el hardware disponible, permite determinar la cantidad de servicios virtualizados que pueden ser prestados en un modelo de infraestructura como servicio. Este paradigma de computación, representa un cambio en la forma en que pueden realizarse las inversiones de hardware de una institución académica; los costos cada vez más altos de centros de cómputo subutilizados, representados en gastos de consumo de energía eléctrica, climatización, adecuación de instalaciones y seguridad física de los equipos, convierten este tipo de proyectos en soluciones de bajo costo, para el uso eficiente de recursos computacionales.

\section{Agradecimientos}

Los autores agradecen a la Fundación Raúl Ocazionez por su interés en la investigación y el apoyo recibido por el grupo CONUSS; además, a la Universidad Industrial de Santander por el apoyo para el desarrollo de proyectos de investigación. Agradecemos también al Grupo de Arquitectura Distribuida de la Universidad Complutense de Madrid por su aporte a la comunidad del software libre, con su 
herramienta OpenNebula. A las comunidades de desarrollo de software libre, por aportar sus conocimientos para el beneficio común.

\section{Referencias}

Ark | Intel ${ }^{\circledR}$ Pentium ${ }^{\circledR}$ III Processor (2012). $667 \mathrm{MHz}, 256 \mathrm{~K}$ Cache, 133 MHz FSB. 2012, Recuperado de

http://ark.intel.com/products/27548/IntelPentium-III-Processor-667-MHz-256K-Cache133-MHz-FSB

Barbosa, A. \& Muñoz, E. (2012). Instalación, Administración, Configuración e Implementación de Servidores Linux con Énfasis en el Desarrollo de un Modelo Administrativo y la Creación de un Prototipo de Clúster de Alta Disponibilidad. Tesis de Pregrado en Ingeniería de Sistemas. Universidad Industrial de Santander. Bucaramanga.

Cloud Computing State of the Union (2011). Disponible en

http://mediasrc.zenoss.com/documents/cloud_c omputing_infographic.pdf

Diaz, E. (2012). Modelo y prototipo de servicios de Computación en la Nube para estudiantes y profesores de la Escuela de Ingeniería de Sistemas e Informática de la Universidad Industrial de Santander. Tesis de Pregrado en Ingeniería de Sistemas. Universidad Industrial de Santander. Bucaramanga.

ESSAC (2012). Tarifas Aplicadas al mes de Febrero de 2012. Recuperado de http://www.essa.com.co/essa/wrm/tariffs_docu
ments/TARIFAS_ESSA_FEBRERO_2012.pdf eXtreme Power Supply Calculator (2012). Extreme Outer Vision. Disponible en http://extreme.outervision.com/PSUEngine

Forrester Research, Incorporated (2011). Is Cloud Computing Ready For The Enterprise? Recuperado de http://www.forrester.com/Research/Document/ Excerpt/0,7211,44229,00.html

Gartner, Incorporated (2011). Gartner Says Cloud Computing Will Be as Influential as Ebusiness. Recuperado de

http://www.gartner.com/it/page.jsp?id=707508.

Geelan, J. (2011). Twenty one experts define cloud computing. Virtualization. Recuperado de http://virtualization.sys-con.com/node/612375.

Maggiani, R. (2011). Cloud computing is changing how we communicate. Professional Communication Conference. Recuperado de $\mathrm{http}: / /$ ieeexplore.ieee.org/stamp/stamp.jsp?tp= \&arnumber $=5208703 \&$ isnumber $=520866$

OCCI (2011). Service Configuration 2.2. Recuperado de

http://opennebula.org/documentation:archives:r el2.2:occicg

Open Cloud Computing Interface - Working Group(C. (2011). Recuperado de http://occiwg.org/

OpenNebula ${ }^{\circledR}$ (2011). The Open Source Solution for Data Center Virtualization. Recuperado de http://opennebula.org 
Peter, D. (2010). A Comparison and Critique of Eucalyptus, OpenNebula and Nimbus. University of Notre Dame. Recuperado de http://www.cse.nd.edu/ ccl/research/papers/pse mpoli-cloudcom.pdf

Schader, M. (2011). Hybrid Clouds: Comparing Cloud Toolkits. Seminar Paper. Chair in Information Systems. University of Mannheim.
VirtualBox ${ }^{\circledR}$ Personal Use and Evaluation License, Oracle (2011). Recuperado de https://www.virtualbox.org/wiki/VirtualBox_P UEL

.

